\title{
Outage analysis of a dual-hop OFDM amplify-and-forward relay system with subcarrier mapping in Rayleigh fading
}

\author{
Raza Ali Shah ${ }^{1 *}$, Nandana Rajatheva ${ }^{2}$ and Yusheng $\mathrm{Ji}^{3,4}$
}

\begin{abstract}
Subcarrier mapping (SCM) is considered to be crucial for capacity-maximization in an orthogonal frequency division multiplexing (OFDM) relaying network. Although SCM in an OFDM system has been investigated extensively, nevertheless, the outage probability and outage capacity analysis of the system is not yet available in the literature. This paper presents the outage analysis of a dual-hop OFDM relay system with ordered subcarrier paring schemes, i.e., worst-to-best (WTB) SCM and best-to-best (BTB) SCM. Accurate close-form expressions are derived for end-to-end outage while considering a dual-hop fixed gain amplify-and-forward (AF) relaying system with Rayleigh fading channel model. The first and second moments of end-to-end signal-to-noise ratio (SNR) and capacity are derived for WTB SCM and BTB SCM schemes while approximating the probability distribution function (PDF) of total capacity of each subcarrier pair by Gaussian distribution. The outage performances of these schemes are compared with balanced links, i.e., when the SNR is same for both hops, as well as unbalanced links, i.e., when the SNR of the second hop is one half of the SNR of the first hop. The numerical results validate the analysis in Rayleigh fading channel.
\end{abstract}

Keywords: Outage probability; Outage capacity; Dual-hop; OFDM; Subcarrier mapping; Rayleigh fading

\section{Introduction}

Orthogonal frequency division multiplexing (OFDM) is the key element of long-term evolution (LTE) while a multi-hop network increases the coverage area of transmission and provides significant performance improvement as a result of cooperative diversity. An OFDM relaying network is, therefore, supported in LTEadvance and is expected to be enhanced in $5 \mathrm{G}$ of wireless communications [1-3]. In a relaying system, the source node transmits data to one or more intermediate nodes. These intermediate nodes or relays retransmit the signal that is received from a source to a destination after some reprocessing. Various types of relays are known in the literature wherein the amplify-and-forward (AF) and decode-and-forward (DF) are most common among the researchers [4]. In an AF relaying system, the relay node amplifies the received signal before forwarding it to the destination node. In a DF relaying system, the received

*Correspondence: rzalsh@gmail.com

${ }^{1}$ Department Telecommunications, AIT, Pathumthani, Thailand

Full list of author information is available at the end of the article signal is decoded at the relay and regenerated before forwarding to the destination node. The performance analysis of these relays is presented in [5]. An AF relay is less complex as compared to a DF relay as it does not require decoding or demodulation of transmitted symbols. In this paper, we focus on the analysis of a two-hop AF relaying system.

Subcarrier mapping (SCM), which is considered to be a capacity-maximization scheme for a dual-hop OFDM relay system, is studied extensively in the literature [6-16]. Two types of ordered SCM schemes, i.e., worst-to-best (WTB) and best-to-best (BTB) are commonly considered. For the purpose of pairing the subcarriers of a sourceto-relay link or hop-1 to the subcarriers of a relay-todestination link or hop-2, the subcarriers of each hop are ordered in ascending/descending order of magnitudes of their channel frequency response (CFR). In WTB SCM, the received signal on a subcarrier of hop-1 with a worst CFR, i.e., the subcarrier with lowest magnitude of CFR is forwarded on the best subcarrier, i.e., the subcarrier with greatest magnitude of CFR, of hop-2. The second worst subcarrier of hop-1 is mapped to the second best

\section{照 Springer}

(C) 2014 Shah et al.: licensee Springer. This is an Open Access article distributed under the terms of the Creative Commons

Attribution License (http://creativecommons.org/licenses/by/4.0), which permits unrestricted use, distribution, and reproduction in any medium, provided the original work is properly credited. 
subcarrier of hop-2 and so on. In BTB SCM, the received signal on subcarrier of hop- 1 with best CFR is forwarded on the best subcarrier of hop- 2 . The second best subcarrier of hop- 1 is mapped to the second best subcarrier of hop- 2 and so on. In particular, the mapping of subcarriers for an OFDM AF relay system is initially introduced in [6]. In [7], numerical analysis for SCM with reduced signaling overhead, by using the subsets of subcarriers, is presented. In [8], close-form expressions for end-to-end signal-tonoise ratio (SNR) and capacity are derived for a downlink dual-hop orthogonal frequency division multiple access (OFDMA) AF relay system by considering Rayleigh fading on hop 2. In [9], close-form capacity analysis is presented by considering Rayleigh fading on both hops. In [10], analysis of a dual-hop OFDM relay system is presented for $\mathrm{AF}$ as well as DF relay. In [11], ergodic capacity comparison is presented for different mapping schemes and relaying protocols. In [12], SCM is studied for the bit-error rate (BER) minimization. In [13], different SCM schemes are compared and close-form expressions are derived for BER and capacity in Rayleigh fading. An interesting recent work on SCM-based opportunistic relaying, presented in $[14,15]$, is worth mentioning. Specifically, [14] presents an optimized solution to minimize the sum power subject to sum rate constraint for the dual-hop OFDM system. In [15], capacity maximization subject to total power constraint is investigated. It is shown that a proposed scheme achieves maximum capacity for various positions of relay in a dual-hop system. Although [14,15] share valuable recent contributions, yet these consider a DF relaying system while this paper focuses on AF relaying. In [16], BER and capacity analysis of an OFDM fixed gain AF relay system with SCM is presented for Nakagami- $m$ fading.

In the literature above, it has been shown that for the dual-hop OFDM relay system, system-capacity can be maximized with a BTB SCM scheme. The BER and ergodic capacity analysis are available for Rayleigh as well as Nakagami- $m$ channel models; however, none of these present performance analysis based on the outage probability and outage capacity of the system.

The outage probability and outage capacity analysis for a single-carrier system provide the basis for analysis of a multicarrier system. The outage analysis for single-carrier and OFDM systems has been studied in the literature. In particular, [17] presents the outage behavior of fundamental relaying protocols, i.e., AF and DF relaying for high SNR. In [18], close-form expressions are derived for the AF as well as DF relaying system for all SNR regimes. In [19], cooperative communication protocol is proposed for multiuser OFDM network while considering relay-assignment based on outage probability analysis for uplink transmission. In [20], outage probability bounds are derived for a multicarrier cooperative AF relaying system. In [21], asymptotic approximation for the upper bound of outage probability for an OFDM system in Rayleigh fading is presented. In [22], the outage probability and outage capacity analysis for a dual-hop OFDM DF relay system is presented while approximating the probability distribution function (PDF) of total capacity by Gaussian distribution for a single-user scenario. In [23], outage probability analysis for an OFDM half-duplex relaying system is presented. In [24], outage probability analysis presented by [22] is extended for a multiuser OFDMA DF relaying system. Although the above literature provides analysis of outage probability and outage capacity for an OFDM system, yet these do not consider ordered SCM schemes in a dual-hop OFDM relaying system which is challenging due to its inherited complexity. In [25], the outage probability and outage capacity analysis is presented while considering ordered SCM; nevertheless, it is limited to the BTB SCM scheme only.

The specific contribution of this paper is the derivations of analytical expressions for WTB SCM schemes such as

- derivation of higher moments of end-to-end SNR for a dual-hop OFDM AF relay system with ordered SCM,

- close-form expression for outage probability,

- close-form approximation for higher moments of channel capacity for a dual-hop OFDM relay system and

- close-form approximation for outage capacity.

Besides the above contribution, a thorough comparison on the basis of outage probability and outage capacity for the dual-hop OFDM relay system with BTB SCM and WTB SCM is presented which is not available in the previous literature. Although the expression for PDF of endto-end SNR for dual-hop OFDM relay system with SCM was derived earlier, yet its cumulative distribution function (CDF) was not known which limited the researchers to analyze the dual-hop system with SCM on the basis of BER and ergodic capacity only. We provide an alternate solution to derive close-form expressions for outage to completely analyze the system performance with ordered SCM schemes. As the outage probability and outage capacity are important performance measuring parameters of a wired/wireless communication system; therefore, in this paper we focus on the performance comparison of the dual-hop OFDM AF relay system with WTB SCM and BTB SCM schemes on the basis of outage probability and outage capacity.

Remark 1: The CFR of each subcarrier of hop-1 and hop- 2 are considered as i.i.d random variables for derivation of close-form expressions. However, for a practical OFDM relay system, adjacent subcarriers possess certain degree of correlation. Therefore, the uncorrelated groups of subcarriers should be determined based on coherence 
bandwidth of channel to carry out block/chunk-based SCM as suggested in $[7,9]$.

The remaining paper is organized as follows. Section 2 introduces the system model. Close-form expressions for outage probability with WTB SCM and BTB SCM are derived in Section 3. Accurate close-form approximation for outage capacity with ordered SCM schemes is presented in Section 4. Section 5 presents the numerical results and discussion. Finally, the paper is concluded in Section 6.

\section{System model}

The system model for the dual-hop OFDM AF relay system is shown in Figure 1 with a source node S, relay node $\mathrm{R}$, and a destination node $\mathrm{D}$. It is assumed that each node is equipped with a single antenna and there is no direct communication between $\mathrm{S}$ and $\mathrm{D}$. Consider $L$ taps channel impulse response (CIR) for hop-j, where $h_{j}(n)$, with $n=0,1, \ldots, L-1$, denotes the $n$th channel tap coefficient and $j \in\{1,2\}$. The CFRs for $N$ OFDM subcarriers of hop $-j$ can be expressed as

$$
\text { Hop } j \text { channel: } H_{j}^{1}, H_{j}^{2}, \ldots, H_{j}^{N}
$$

where $H_{j}^{k}$ denotes the CFR for $k$ th OFDM subcarrier of hop- $j$ and $k \in\{1,2, \ldots, N\} . H_{j}^{k}$ is assumed to be known at relay. A fixed gain relay $R$ receives the signal that is transmitted from source $S$ in the first time slot and retransmits an amplified signal to destination $\mathrm{D}$ in the second time slot after performing SCM.

Subcarrier mapping: The OFDM relay performs FFT/IFFT operations for mapping the subcarriers to achieve WTB SCM or BTB SCM configuration. For hop-1, consider an ordered set $\left\{\mathcal{S}_{k}: k \in\{1, \ldots, N\}\right\}$ of indexes such that CFR of subcarrier with index $\mathcal{S}_{k+1}$ is better than CFR of subcarrier with index $\mathcal{S}_{k}$. Similarly, for hop-2, consider an ordered set $\left\{\mathcal{D}_{k}: k \in\{1, \ldots, N\}\right\}$ of indexes such that CFR of subcarrier with index $\mathcal{D}_{k}$ is better than CFR of subcarrier with index $\mathcal{D}_{k+1}$. A oneto-one mapping of $S_{k}$ and $D_{k}$, i.e., $S_{k} \rightarrow D_{k}$ results in a WTB SCM scheme, while the mapping $S_{N-k+1} \rightarrow D_{k}$ results in a BTB SCM scheme. For notation simplicity, we drop $\mathcal{S}$ and $\mathcal{D}$ and use the subscript $k$ only when referring to the $k$ th subcarrier pair in the context of WTB or BTB mapping.

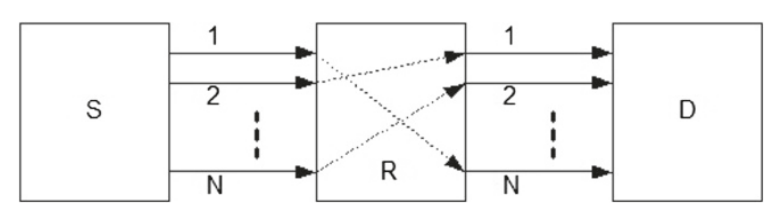

Figure 1 Dual-hop OFDM relay system.
As it is not possible to carry out the analysis for the dual-hop OFDM relay system without imposing certain assumptions on source-to-relay (S-R) and relay-todestination (R-D) links, we, therefore, consider same assumptions as in $[8,9,13]$. The CFRs of subcarriers for both hops are assumed to be independent and identically distributed random variables. This assumption gives exact results for a full-diversity OFDM system, i.e, when $L=N$ and provides approximate analysis for large $L$, i.e., when $L \geq 6$ for the dual-hop OFDM relay system with SCM [16]. The CDF and PDF of SNR for $k$ th subcarrier of hop- $j$ can be expressed as $F_{\gamma_{j}^{k}}(x)=1-e^{-\lambda_{j} x}$ and $f_{\gamma_{j}^{k}}(x)=\lambda_{j} e^{-\lambda_{j} x}$ respectively where $\lambda_{j}=\frac{1}{\bar{\gamma}_{j}}$. Symbol $\bar{\gamma}_{j}=\mathcal{E}_{j} \mathbf{E}\left(\left|H_{j}^{k}\right|^{2}\right) / \sigma_{n_{j}}^{2}$ defines the average SNR of hop- $j$ where $\mathcal{E}_{j}$ and $\left|H_{j}^{k}\right|$ define average symbol power and CFR for $k$ th subcarrier of hop-j. $\mathbf{E}(*)$ denotes the expectation operation. Symbol $\sigma_{n_{j}}^{2}$ represents noise variance of additive white Gaussian noise for $k$ th subcarrier of hop-j. For $k$ th subcarrier pair, the end-to-end SNR for the dual-hop OFDM AF fixed gain relay system with SCM, denoted by $\gamma_{\text {end }}^{k}$, can be expressed as [13]

$$
\gamma_{\mathrm{end}}^{k}=\frac{\gamma_{1}^{k} \gamma_{2}^{k}}{\gamma_{2}^{k}+\epsilon}
$$

where $\gamma_{j}^{k}$ represents the SNR of hop- $j$ for $k$ th subcarrier and constant $\epsilon=\frac{\mathcal{E}_{1}}{G^{2} \sigma_{n_{j}}^{2}}$ depends on relay gain $G[5,13]$. Using the knowledge of order statistics, the PDF $\gamma_{1}^{k}$ for $k$ th weakest subcarrier can be expressed as [13]

$$
f_{\gamma_{1}^{k}}^{w}(\gamma)=\sum_{i=0}^{k-1} \alpha_{i} \lambda_{1} e^{-\rho_{i} \gamma} .
$$

where $\alpha_{i}=N\left(\begin{array}{c}N-1 \\ k-1\end{array}\right)\left(\begin{array}{c}k-1 \\ i\end{array}\right)(-1)^{k-i-1}$ and $\rho_{i}=\lambda_{1}(N-i)$. Similarly, the PDF of $\gamma_{2}^{k}$ for $k$ th strongest subcarrier can be expressed as

$$
f_{\gamma_{2}^{k}}^{s}(\gamma)=\sum_{d=0}^{N-k} \alpha_{d} \lambda_{2} e^{-\rho_{d} \gamma} .
$$

where $\alpha_{d}=N\left(\begin{array}{c}N-1 \\ k-1\end{array}\right)\left(\begin{array}{c}N-k \\ d\end{array}\right)(-1)^{N-k-d}$ and $\rho_{d}=\lambda_{2}(N-d)$. The PDF $f_{\gamma_{1}^{k}}^{s}(\gamma)$ for $k$ th strongest subcarrier of hop- 1 can be obtained as (4). Equations (3) and (4) can be used to derive the close-form expressions for outage probability and outage capacity analysis.

\section{Outage probability}

Outage probability is defined as the probability that instantaneous end-to-end SNR falls below certain predetermined threshold value, i.e., $\gamma_{\text {th }}$. Therefore, the outage 
probability for $k$ th subcarrier pair can be expressed as

$$
P_{\text {out }}^{k}=\operatorname{Pr}\left(\gamma_{\text {end }}^{k} \leq \gamma_{\text {th }}\right)=\operatorname{Pr}\left(\frac{\gamma_{1}^{k} \gamma_{2}^{k}}{\gamma_{2}^{k}+\epsilon} \leq \gamma_{\text {th }}\right)
$$

Equation (5) can be simplified to get a close-form expression for outage probability in terms of CDF of $\gamma_{1}^{k}$ and PDF of $\gamma_{2}^{k}$ which upon further simplification results in the following propositions.

Proposition 1. The close-form expression for end-toend outage probability with WTB SCM, i.e., $\left(P_{\text {out }}^{k}\right)^{\text {WTB }}$ can be expressed as

$$
\begin{aligned}
\left(P_{\text {out }}^{k}\right)^{\mathrm{WTB}}= & 1-\sum_{d=0}^{N-k} \alpha_{d} \frac{1}{N-d} e^{-\rho_{d} \gamma_{\text {th }}}+\sum_{i=0}^{k-1} \sum_{d=0}^{N-k} \alpha_{i} \alpha_{d} \frac{\lambda_{2}}{N-i} e^{-\rho_{d} \gamma_{\text {th }}} \\
& \times\left[\frac{1}{\rho_{d}}-2 \sqrt{\frac{\rho_{i} \epsilon \gamma_{\text {th }}}{\rho_{d}}} K_{1}\left(2 \sqrt{\rho_{i} \rho_{d} \epsilon \gamma_{\text {th }}}\right)\right]
\end{aligned}
$$

\section{Proof. See Appendix A.}

Proposition 2. The close-form expression for end-toend outage probability with BTB SCM, i.e., $\left(P_{\text {out }}^{k}\right)^{\text {BTB }}$ can be derived as

$$
\begin{aligned}
\left(P_{\text {out }}^{k}\right)^{\text {BTB }}= & 1-\sum_{d=0}^{N-k} \alpha_{d} \frac{1}{N-d} e^{-\rho_{d} \gamma_{\text {th }}}+\sum_{i=0}^{N-k} \sum_{d=0}^{N-k} \alpha_{i}^{\prime} \alpha_{d} \frac{\lambda_{2}}{N-i} e^{-\rho_{d} \gamma_{\text {th }}} \\
& \times\left[\frac{1}{\rho_{d}}-2 \sqrt{\frac{\rho_{i} \in \gamma_{\text {th }}}{\rho_{d}}} K_{1}\left(2 \sqrt{\rho_{i} \rho_{d} \in \gamma_{\text {th }}}\right)\right]
\end{aligned}
$$

where $\alpha_{i}^{\prime}=N\left(\begin{array}{c}N-1 \\ k-1\end{array}\right)\left(\begin{array}{c}N-k \\ i\end{array}\right)(-1)^{N-k-i}$.

Proof. See Appendix A.

\section{Outage capacity analysis}

The $\mathrm{q} \%$ outage capacity $\left(C_{q}\right)$ is defined as the transmission rate that is guaranteed for $1-\mathrm{q} / 100$ of channel realizations $[26,27]$. Considering Gaussian distribution for the channel capacity $\left(C^{k}\right)$, the close-form expression for outage capacity, i.e., $C_{q}^{k}$ can be derived. For the $k$ th subcarrier pair, close-form expression for outage capacity can be expressed as

$$
C_{q}^{k}=\mathbf{E}\left[C^{k}\right]+\sqrt{2\left(\mathbf{E}\left[\left(C^{k}\right)^{2}\right]-\left(\mathbf{E}\left[C^{k}\right]\right)^{2}\right)} \operatorname{erfc}^{-1}\left(2-\frac{q}{50}\right)
$$

where $\mathrm{erfc}^{-1}$ denotes the inverse of complementary error function. $\mathbf{E}\left[C^{k}\right]$ and $\mathbf{E}\left[\left(C^{k}\right)^{2}\right]$ denote the first and second moments of channel capacity for $k$ th subcarrier pair respectively and $C^{k}=\frac{1}{2} \log _{2}\left(1+\gamma_{\text {end }}^{k}\right)$. The exact solution of $\mathbf{E}\left[C^{k}\right]$ and $\mathbf{E}\left[\left(C^{k}\right)^{2}\right]$, hence, (8) is not known in the literature; nevertheless, the second-order approximation of ergodic capacity can be expressed in the form of first and second moments of $\gamma_{\mathrm{end}}^{k}$ as [26,28],

$\mathbf{E}\left[C^{k}\right] \approx \frac{1}{2} \log _{2} e\left[\ln \left(1+\mathbf{E}\left[\gamma_{\text {end }}^{k}\right]\right)-\frac{\mathbf{E}\left[\left(\gamma_{\text {end }}^{k}\right)^{2}\right]-\left(\mathbf{E}\left[\gamma_{\text {end }}^{k}\right]\right)^{2}}{2\left(1+\mathbf{E}\left[\gamma_{\text {end }}^{k}\right]\right)^{2}}\right]$

and

$$
\begin{aligned}
\mathbf{E}\left[\left(C^{k}\right)^{2}\right] \approx & \frac{1}{4}\left(\log _{2} e\right)^{2}\left[\left(\ln \left(1+\mathbf{E}\left[\gamma_{\text {end }}^{k}\right]\right)\right)^{2}\right. \\
& +\frac{\mathbf{E}\left[\left(\gamma_{\text {end }}^{k}\right)^{2}\right]-\left(\mathbf{E}\left[\gamma_{\text {end }}^{k}\right]\right)^{2}}{\left(1+\mathbf{E}\left[\gamma_{\text {end }}^{k}\right]\right)^{2}} \\
& \left.\times \ln \left(\frac{e}{1+\mathbf{E}\left[\gamma_{\text {end }}^{k}\right]}\right)\right]
\end{aligned}
$$

respectively, where $e$ is the Neper's number. Equations (9) and (10) can be used to solve (8). It remains to find $\mathbf{E}\left[\gamma_{\text {end }}^{k}\right]$ and $\mathbf{E}\left[\left(\gamma_{\text {end }}^{k}\right)^{2}\right]$ for WTB SCM and BTB SCM schemes.

Proposition 3. The close-form expression for q\% outage capacity with WTB SCM scheme can be expressed in compact form as

$$
\begin{aligned}
\left(C_{q}^{k}\right)^{\mathrm{WTB}}= & \mathbf{E}^{\mathrm{WTB}}\left[C^{k}\right]+\sqrt{2\left(\mathbf{E}^{\mathrm{WTB}}\left[\left(C^{k}\right)^{2}\right]-\left(\mathbf{E}^{\mathrm{WTB}}\left[C^{k}\right]\right)^{2}\right)} \\
& \times \operatorname{erfc}^{-1}\left(2-\frac{q}{50}\right)
\end{aligned}
$$

where $\mathbf{E}^{\text {WTB }}\left[C^{k}\right]$ and $\mathbf{E}^{\text {WTB }}\left[\left(C^{k}\right)^{2}\right]$ represent the first and second moments of ergodic capacity for WTB SCM, respectively.

\section{Proof. See Appendix B.}

Proposition 4. The close-form expression for q\% outage capacity with BTB SCM scheme can be expressed in compact form as

$$
\begin{aligned}
\left(C_{q}^{k}\right)^{\mathrm{BTB}}= & \mathbf{E}^{\mathrm{BTB}}\left[C^{k}\right]+\sqrt{2\left(\mathbf{E}^{\mathrm{BTB}}\left[\left(C^{k}\right)^{2}\right]-\left(\mathbf{E}^{\mathrm{BTB}}\left[C^{k}\right]\right)^{2}\right)} \\
& \times \operatorname{erfc}^{-1}\left(2-\frac{q}{50}\right)
\end{aligned}
$$


where $\mathbf{E}^{\mathrm{BTB}}\left[C^{k}\right]$ and $\mathbf{E}^{\mathrm{BTB}}\left[\left(C^{k}\right)^{2}\right]$ represent the first and second moments of ergodic capacity for BTB SCM, respectively.

Proof. See Appendix C.

\section{Numerical results and discussion}

This section presents numerical results of outage probability and outage capacity for the dual-hop OFDM AF relay system with ordered SCM schemes in Rayleigh fading. Although the derived expressions compute the outage for any values of $\bar{\gamma}_{1}$ and $\bar{\gamma}_{2}$, we limit the discussion to two special cases, i.e., the balanced links case $\left(\bar{\gamma}_{1}=\bar{\gamma}_{2}\right.$, i.e., $\left.\lambda_{1}=\lambda_{2}\right)$ and the unbalanced links case $\left(\bar{\gamma}_{1}=2 \bar{\gamma}_{2}\right.$, i.e., $2 \lambda_{1}=\lambda_{2}$ ). Numerical results for each figure are obtained by considering $10^{6}$ channel realizations. The threshold, i.e., $\gamma_{\text {th }}$ is set to $0 \mathrm{~dB}$ while $N=16$.

\subsection{Outage probability}

To describe the outage probability, three subsets, i.e., $\mathbf{B}=$ $\left\{k: k \in\{1, \ldots, N\} \wedge P_{\text {out }}^{k} \leq 0.1, \forall k\right\}, \mathbf{M}=\{k: k \in$ $\left.\{1, \ldots, N\} \wedge 0.1<P_{\text {out }}^{k}<0.9, \forall k\right\}$, and $\mathbf{P}=\{k: k \in$ $\left.\{1, \ldots, N\} \wedge P_{\text {out }}^{k} \geq 0.9, \forall k\right\}$ of indexes are defined as best, moderate, and poor subsets of subcarrier pairs, respectively. Similarly, low, medium, and high SNR regimes are defined as $\mathrm{SNR} \leq 6 \mathrm{~dB}, 6 \mathrm{~dB}<\mathrm{SNR}<12 \mathrm{~dB}$ and $\mathrm{SNR} \geq$ $12 \mathrm{~dB}$, respectively. The performance of system with $\mathrm{BTB}$ SCM and WTB SCM is compared for two special cases, i.e., $\bar{\gamma}_{1}=\bar{\gamma}_{2}$ and $\bar{\gamma}_{1}=2 \bar{\gamma}_{2}$.

\subsubsection{Special case 1: balanced links $\left(\bar{\gamma}_{1}=\bar{\gamma}_{2} ; \lambda_{1}=\lambda_{2}\right)$}

Figure 2 presents numerical results for outage probability with a BTB SCM scheme and $\bar{\gamma}_{1}=\bar{\gamma}_{2}$. The analytical results are computed using (7). The subcarrier pair with index $k=1$ refers to the best subcarrier pair which maps the best subcarrier of hop-1 to the best subcarrier of hop2 . Similarly $k=2$ refers to the second best pair and so on. For $\bar{\gamma}_{1}=\bar{\gamma}_{2}=4 \mathrm{~dB}$, the subsets $\mathbf{B}=\{1, \ldots, 5\}$, $\mathbf{M}=\{6, \ldots, 9\}$ and $\mathbf{P}=\{10, \ldots, 16\}$ can be updated from Figure 2 . These share $31 \%, 25 \%$, and $44 \%$ of total subcarrier pairs, respectively. High outage can be observed in a low SNR regime as the subset $\mathbf{P}$ outnumber $\mathbf{B}$ and $\mathbf{M}$. For $\bar{\gamma}_{1}=\bar{\gamma}_{2}=8 \mathrm{~dB}$, these subsets can be updated as $\mathbf{B}=\{1, \ldots, 9\}, \mathbf{M}=\{10, \ldots, 13\}$, and $\mathbf{P}=\{14, \ldots, 16\}$ which share $56 \%, 25 \%$, and $19 \%$ of total pairs, respectively. It shows that the number of high reliable pairs increases in a medium SNR regime as $\mathbf{B}$ outnumber $\mathbf{M}$ and $\mathbf{P}$. For $\bar{\gamma}_{1}=$ $\bar{\gamma}_{2}=12 \mathrm{~dB}$, the subsets $\mathbf{B}=\{1, \ldots, 12\}, \mathbf{M}=\{13,14\}$, and $\mathbf{P}=\{15, \ldots, 16\}$ share $75 \%, 13 \%$, and $12 \%$ of total subcarriers, respectively. Hence, the number of pairs in outage decreases further as only $12 \%$ of total pairs belong to $\mathbf{P}$.

It is worth mentioning that BTB SCM makes it possible to identify subcarrier pairs with lower outage probability or high reliability even at low SNR. Although the subcarriers of each hop experience different channel fading, yet sorting on the basis of fading then mapping in such a way that lower faded subcarrier of hop-1 mapped to lower faded subcarrier of hop-2

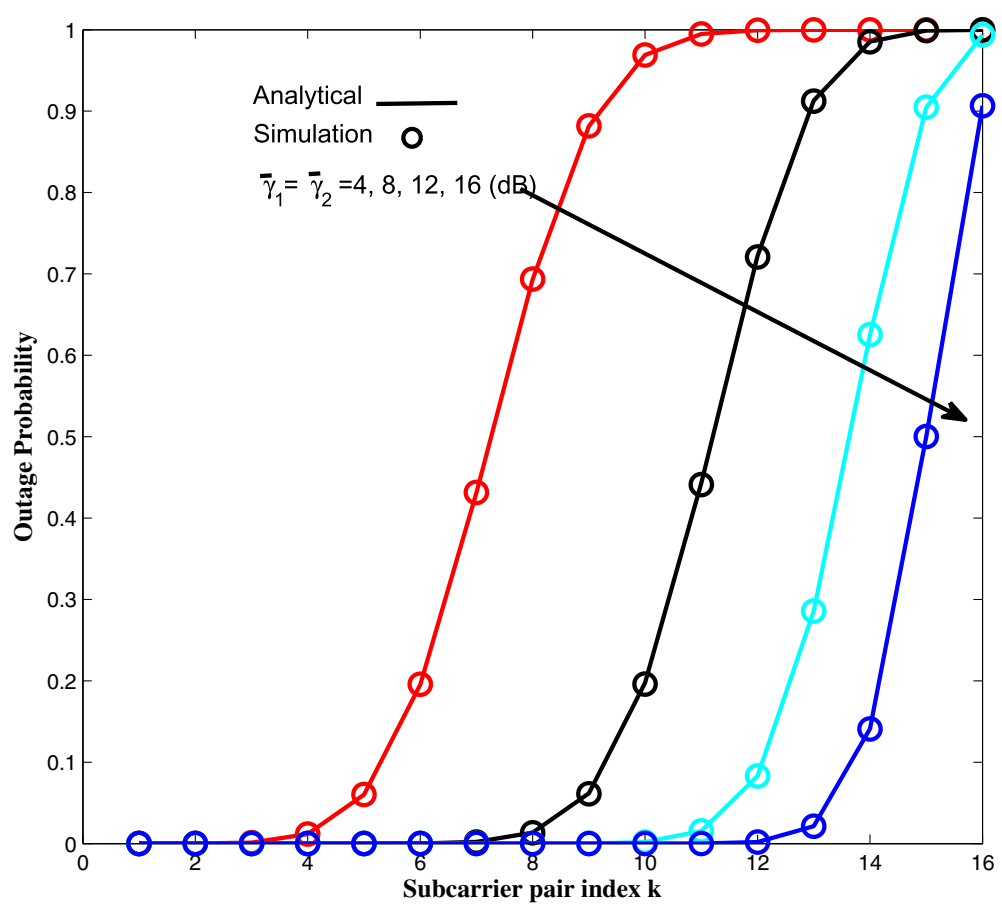

Figure 2 BTB SCM: Outage probability for the $k$ th best subcarrier pair, $\bar{\gamma}_{1}=\bar{\gamma}_{2}$. 
yields a large number of reliable end-to-end subcarrier pairs.

Figure 3 depicts numerical results for outage probability with the WTB SCM scheme and $\bar{\gamma}_{1}=\bar{\gamma}_{2}$. The analytical results are computed using (6). The subcarrier pair with index $k=1$ refers to the pair with worst subcarrier of hop-1 and best subcarrier of hop-2. Similarly, $k=2$ refers to a subcarrier pair with the second worst subcarrier of hop-1 mapped to the second best subcarrier of hop2 and so on. For $\bar{\gamma}_{1}=\bar{\gamma}_{2}=4 \mathrm{~dB}$, the subsets $\mathbf{B}=\{\}$, $\mathbf{M}=\{1, \ldots, 10\}$ and $\mathbf{P}=\{11, \ldots, 16\}$ share $0 \%, 62 \%$, and $38 \%$ of total subcarrier pairs. The system experiences high outage in the low SNR regime as it is mostly dominated by moderate and poor subsets of subcarrier pairs. For $\bar{\gamma}_{1}=\bar{\gamma}_{2}=8 \mathrm{~dB}$, the subsets $\mathbf{B}=\{3, \ldots, 10\}, \mathbf{M}=$ $\{1,2,11,12, \ldots, 15\}$, and $\mathbf{P}=\{16\}$ share $50 \%, 44 \%$, and $6 \%$ of total subcarrier pairs, respectively. The outage performance is dominated by best and moderate subsets in this regime. For $\bar{\gamma}_{1}=\bar{\gamma}_{2}=12 \mathrm{~dB}$, the subsets $\mathbf{B}=\{1, \ldots, 13\}$, $\mathbf{M}=\{14,15,16\}$, and $\mathbf{P}=\{\}$ share $81 \%, 19 \%$, and $0 \%$ of total subcarrier pairs, respectively. Interestingly, there is no poor pair for the WTB SCM scheme in the high SNR regime. For any $k$, the variation of outage probability with $\bar{\gamma}_{1}$ is worth noting, e.g., for $k=1$ the outage probability is $0.87,0.41,0.13$, and 0.04 at $\bar{\gamma}_{1}=\bar{\gamma}_{2}=4,8,12,16 \mathrm{~dB}$, respectively.

It should be noted that, for WTB SCM, the pairs with lower outage probability or high reliability can only be identified in the medium and high SNR regimes. At low SNR, e.g., $\bar{\gamma}_{1}=\bar{\gamma}_{2}=4 \mathrm{~dB}$, highly reliable links can not be identified. The reason is mapping of hop-1's subcarrier with the lowest channel fading to the subcarrier of hop2 that experience the highest channel fading and so on. It reduces the number of end-to-end reliable subcarrier pairs in low SNR regime.

\subsubsection{Special Case 2: Unbalanced links $\left(\bar{\gamma}_{1}=2 \bar{\gamma}_{2} ; 2 \lambda_{1}=\lambda_{2}\right)$}

Figure 4 presents numerical results for outage probability with BTB SCM and $\bar{\gamma}_{1}=2 \bar{\gamma}_{2}$, i.e., $2 \lambda_{1}=\lambda_{2}$. It should be noted that theoretical results for any values of $\bar{\gamma}_{1}$ and/or $\bar{\gamma}_{2}$ can be computed using (6) and/or (7). Higher outage can be observed for $\bar{\gamma}_{1}=2 \bar{\gamma}_{2}$ by comparing Figure 2 and Figure 4, e.g., for $k=5$ the outage probability is 0.03 in Figure 2 which increases to 0.38 in Figure 4 . The reason for this increase is $\bar{\gamma}_{2}$ which equals one half of $\bar{\gamma}_{1}$ in Figure 4. For $\bar{\gamma}_{1}=2 \bar{\gamma}_{2}=4 \mathrm{~dB}$, the subsets $\mathbf{B}=\{1, \ldots, 3\}$, $\mathbf{M}=\{4, \ldots 7\}$, and $\mathbf{P}=\{8, \ldots, 16\}$ share $25 \%, 19 \%$, and $56 \%$ of total subcarrier pairs, respectively. The outage performance in low SNR regime is dominated by worst subcarrier pairs as $\mathbf{P}$ share highest percentage of total pairs. For $\bar{\gamma}_{1}=2 \bar{\gamma}_{2}=8 \mathrm{~dB}$, the subsets $\mathbf{B}=\{1, \ldots, 6\}$, $\mathbf{M}=\{7, \ldots 10\}$, and $\mathbf{P}=\{11, \ldots, 16\}$ share $38 \%, 24 \%$, and $38 \%$ of total subcarrier pairs, respectively. The outage reduces significantly in this regime as compared to the low SNR. For $\bar{\gamma}_{1}=2 \bar{\gamma}_{2}=12 \mathrm{~dB}$, the subsets $\mathbf{B}=\{1, \ldots, 8\}$,

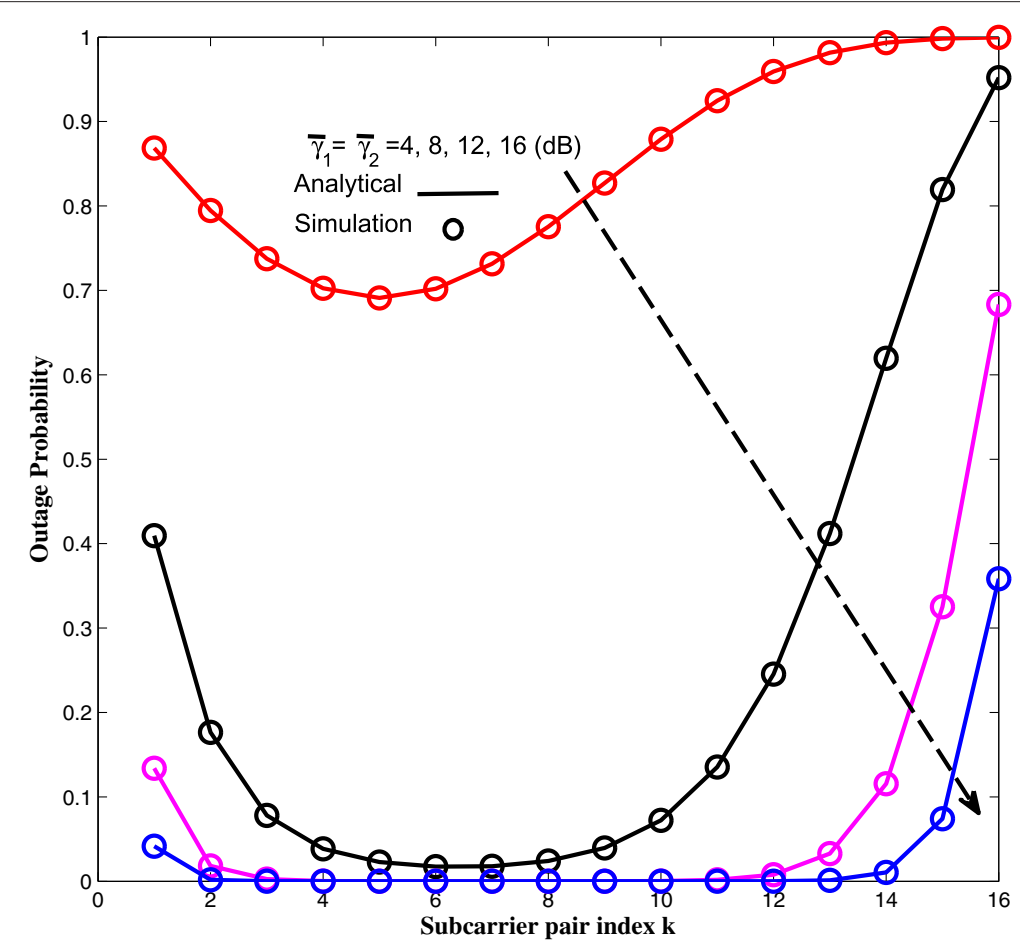

Figure 3 WTB SCM: Outage probability for the $k$ th subcarrier pair, $\bar{\gamma}_{1}=\bar{\gamma}_{2}$. 


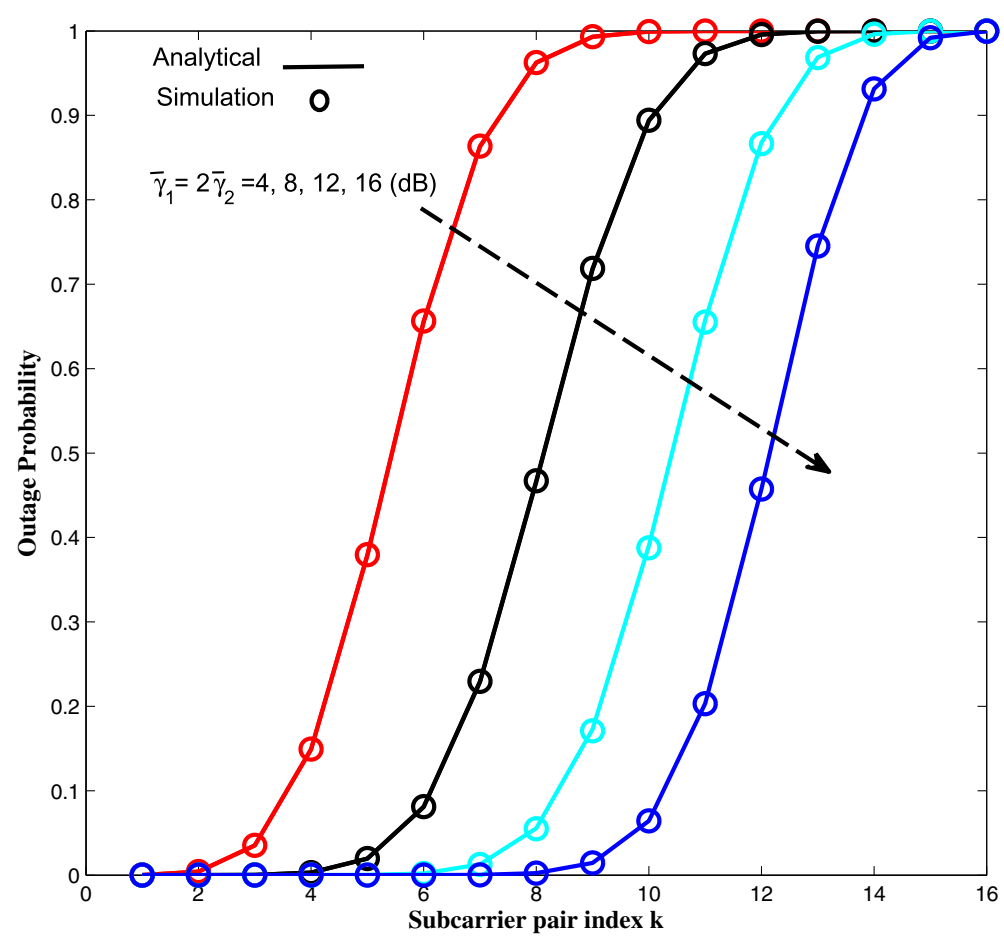

Figure 4 BTB SCM: Outage probability for the $k$ th best subcarrier pair, $\bar{\gamma}_{1}=2 \bar{\gamma}_{2}$.

$\mathbf{M}=\{9, \ldots 12\}$, and $\mathbf{P}=\{13, \ldots, 16\}$ share $50 \%, 25 \%$, and $25 \%$ of total subcarrier pairs, respectively. Significant reduction in the outage can be observed in this regime as compared to medium SNR.

Figure 5 depicts numerical results for outage probability with WTB SCM and $\bar{\gamma}_{1}=2 \bar{\gamma}_{2}$. Higher outage can be observed for any $k$ in case of $\bar{\gamma}_{1}=2 \bar{\gamma}_{2}$ as compared to $\bar{\gamma}_{1}=\bar{\gamma}_{2}$ scenario due to lower $\bar{\gamma}_{2}$. For $\bar{\gamma}_{1}=2 \bar{\gamma}_{2}=$ $4 \mathrm{~dB}$, the two subsets $\mathbf{B}$ and $\mathbf{M}$ are empty. Highest outage can be observed in this SNR regime as all the subcarrier pairs belong to $\mathbf{P}$. For $\bar{\gamma}_{1}=2 \bar{\gamma}_{2}=8 \mathrm{~dB}$, the subsets $\mathbf{M}=\{1, \ldots 11\}$ and $\mathbf{P}=\{12, \ldots, 16\}$ share $69 \%$ and $31 \%$ of total subcarrier pairs, respectively. The outage is dominated by moderate subcarrier pairs in this regime. For $\bar{\gamma}_{1}=2 \bar{\gamma}_{2}=12 \mathrm{~dB}$, the subsets $\mathbf{B}=\{4, \ldots, 8\}, \mathbf{M}=$ $\{1, \ldots, 3,9, \ldots 14\}$, and $\mathbf{P}=\{15,16\}$ share $31 \%, 56 \%$, and $13 \%$ of total subcarrier pairs, respectively, i.e., outage in this regime is dominated by the moderate subcarrier pairs.

\subsubsection{Variation of outage probability with SNR}

This subsection describes special case 2 further for a wide range of SNR. The variation of outage probability with the BTB SCM scheme is depicted for $\bar{\gamma}_{1}=2 \bar{\gamma}_{2} \in$ $\{0,2, \ldots, 20\} \mathrm{dB}$ in Figure 6. Two subcarrier pairs are selected from the best, moderate, and poor subsets for performance comparison. From $\mathbf{B}$, the pairs $k=2,4$ are selected which experience lower channel fading as compared to other subcarrier pairs. These pairs have low outage probability even in the low SNR regime. Their outage probability approaches $10^{-2}$ for $\bar{\gamma}_{1}=4.2 \mathrm{~dB}$ and $\bar{\gamma}_{1}=6.6 \mathrm{~dB}$, respectively. The pairs $k=7,9$ are selected from $\mathbf{M}$. These achieve outage probability of $10^{-2}$ at $\bar{\gamma}_{1}=$ $9.5 \mathrm{~dB}$ and $\bar{\gamma}_{1}=12 \mathrm{~dB}$, respectively. The subcarrier pairs $k=12,13$ are selected from $\mathbf{P}$. These show high outage probability at low and medium SNR. Their outage probability approaches $10^{-2}$ at $\bar{\gamma}_{1}=16.8 \mathrm{~dB}$ and $\bar{\gamma}_{1}=19.5 \mathrm{~dB}$, respectively.

Variation of outage probability with the WTB SCM scheme is depicted for $\bar{\gamma}_{1}=2 \bar{\gamma}_{2} \in\{0,2, \ldots, 20\} \mathrm{dB}$ in Figure 7. The subcarrier pairs $k=7,5$ are selected from $\mathbf{B}$. These pairs achieve $10^{-2}$ outage probability at $\bar{\gamma}_{1}=10.8$ $\mathrm{dB}$ and $\bar{\gamma}_{1}=11 \mathrm{~dB}$, respectively. The subcarrier pairs $k=11,2$ are selected from $\mathbf{M}$ and achieve $10^{-2}$ outage probability at $\bar{\gamma}_{1}=13.2 \mathrm{~dB}$ and $\bar{\gamma}_{1}=15.2 \mathrm{~dB}$, respectively. The subcarrier pairs $k=14,15$ are selected from P. The pair $k=14$ achieve $10^{-2}$ outage probability at $\bar{\gamma}_{1}=19 \mathrm{~dB}$.

Figures 6 and 7 lead to important conclusion, i.e., the BTB SCM scheme outperforms the WTB SCM scheme on the basis of outage probability performance for the best and moderated subcarrier pairs, e.g., $k=$ 2, 4,7,9 in Figure 6 and $k=7,5,11,2$ in Figure 7 . The performance of poor pairs $k=12,13$ and $k=$ 14,15 is approximately similar for both schemes as these show high outage probability except in the high SNR regime. 


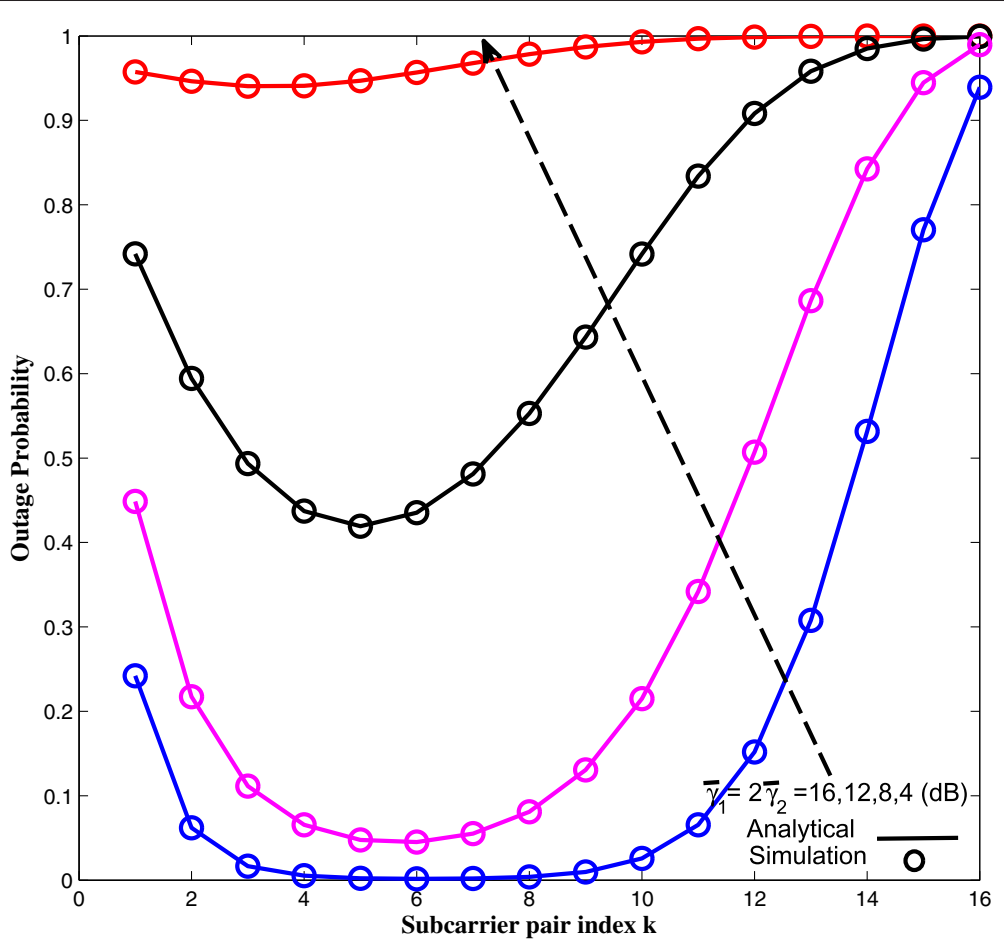

Figure 5 WTB SCM: Outage probability for the $k$ th subcarrier pair, $\bar{\gamma}_{1}=2 \bar{\gamma}_{2}$.

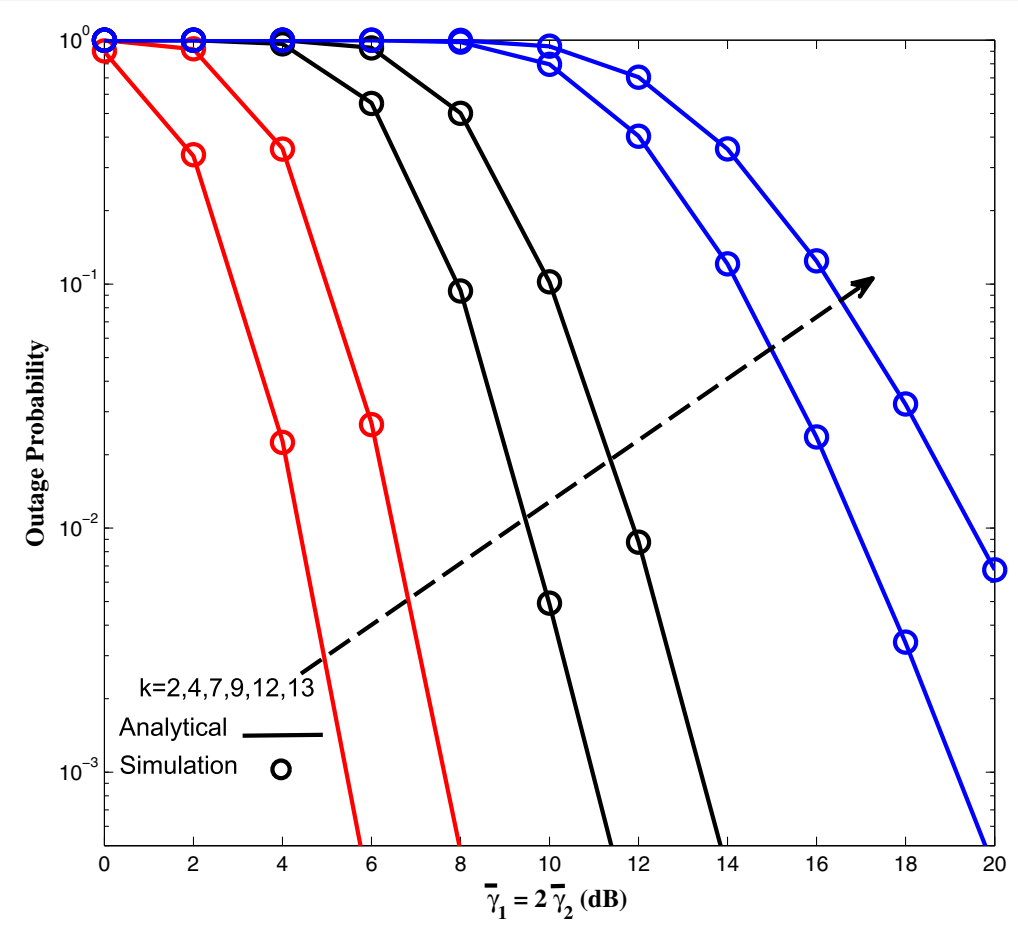

Figure 6 BTB SCM: Variation of outage probability for unbalanced link, $\bar{\gamma}_{1}=2 \bar{\gamma}_{2}$. 


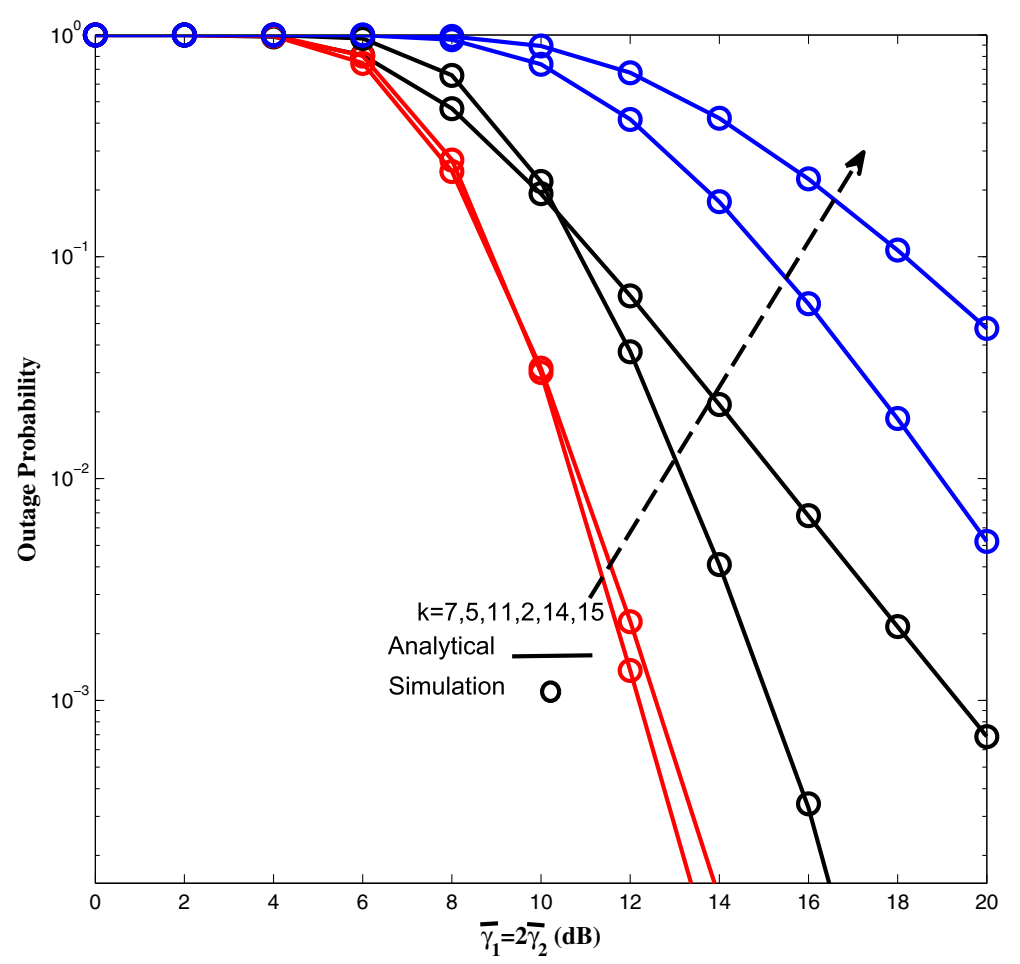

Figure 7 WTB SCM: Variation of outage probability for unbalanced link, $\bar{\gamma}_{1}=2 \bar{\gamma}_{2}$.

\subsection{Outage capacity}

Figure 8 depicts theoretical and simulation results for $10 \%$ outage capacity with the BTB SCM scheme for $k=1,5,8,12,14,15$ while $\bar{\gamma}_{1}=\bar{\gamma}_{2}$ and $\bar{\gamma}_{1}=2 \bar{\gamma}_{2}$. The theoretical results are obtained using (12). Considering 1 $\mathrm{bit} / \mathrm{sec} / \mathrm{Hz}$ as the threshold, it can be observed that, for $\bar{\gamma}_{1}=\bar{\gamma}_{2}$, the pairs $k=1,5$ achieve the threshold at $\bar{\gamma}_{1}=1.5 \mathrm{~dB}$ and $\bar{\gamma}_{1}=6 \mathrm{~dB}$, respectively. These subcarrier pairs are selected from $\mathbf{B}$ and contribute to higher capacity even in low SNR regime as compared to other pairs. The subcarrier pairs $k=8,12$ are selected from $\mathbf{M}$. These achieve unity threshold at $\bar{\gamma}_{1}=8.3 \mathrm{~dB}$ and $\bar{\gamma}_{1}=12.8 \mathrm{~dB}$, respectively. The subcarrier pairs $k=14,15$ are selected from $\mathbf{P}$. These achieve the threshold at $\bar{\gamma}_{1}=16.5 \mathrm{~dB}$ and $\bar{\gamma}_{1}=20.1 \mathrm{~dB}$, respectively. It can be observed that for any $\bar{\gamma}_{1}$, higher value of $k$ corresponds to a lower outage capacity. It is according to the theory as the subcarrier pair with index $k-1$ is better than the subcarrier pair $k$ for BTB SCM. A similar trend can be observed for $\bar{\gamma}_{1}=2 \bar{\gamma}_{2}$. It is worth mentioning that, for any $\bar{\gamma}_{1}$, the outage capacity of any $k$ is slightly lower for $\bar{\gamma}_{1}=2 \bar{\gamma}_{2}$ than its value for $\bar{\gamma}_{1}=\bar{\gamma}_{2}$. This difference of capacities for any $k$ decreases as $k \rightarrow 1$ and increases as $k \rightarrow 16$. Specifically, at SNR $=20 \mathrm{~dB}$, the difference of capacities for $\bar{\gamma}_{1}=\bar{\gamma}_{2}$ and $\bar{\gamma}_{1}=2 \bar{\gamma}_{2}$ is much less for $k=1$ as compared to $k=15$.

Figure 9 depicts theoretical and simulation results for $10 \%$ outage capacity of subcarrier pairs $k=1,2,4,5,10,14$ for the WTB SCM scheme. The theoretical results are obtained using (11). It can be observed that unity threshold is achieved by $k=14,10$ at $\bar{\gamma}_{1}=8.5 \mathrm{~dB}$. These are selected from $\mathbf{B}$ for $\bar{\gamma}_{1}=\bar{\gamma}_{2}$ and contribute to higher outage capacity in medium and high SNR regimes as compared to other subcarrier pairs. The outage capacity of these pairs approaches $5 \mathrm{Bits} / \mathrm{sec} / \mathrm{Hz}$ at $\bar{\gamma}_{1}=30 \mathrm{~dB}$. For $\bar{\gamma}_{1}=2 \bar{\gamma}_{2}$, a slightly lower outage capacity can be observed for these pairs. The threshold is achieved at $\bar{\gamma}_{1}=2 \bar{\gamma}_{2}=10 \mathrm{~dB}$ by these pairs. Hence, $1.5 \mathrm{~dB}$ SNR degradation is observed in the case of unbalanced links. A similar effect can be observed for all subcarrier pairs, e.g., for $k=14$, approximately $1 \mathrm{~dB}$ SNR degradation can be observed at $4.5 \mathrm{Bits} / \mathrm{sec} / \mathrm{Hz}$ due to unbalanced links. From $\mathbf{M}$, the selected pairs $k=5,4$ achieve threshold at $11 \mathrm{~dB}$ and $12 \mathrm{~dB}$ respectively for $\bar{\gamma}_{1}=\bar{\gamma}_{2}$. From $\mathbf{P}$, the selected pairs $k=2,1$ achieve threshold at $15 \mathrm{~dB}$ and 19 $\mathrm{dB}$, respectively for $\bar{\gamma}_{1}=\bar{\gamma}_{2}$. It is worth mentioning that, although for any $k$, the outage capacity in case of $\bar{\gamma}_{1}=\bar{\gamma}_{2}$ is greater than $\bar{\gamma}_{1}=2 \bar{\gamma}_{2}$, yet unlike BTB SCM, this difference is more prominent in best subcarrier pairs than the poor ones, e.g., by observing the plots for $\bar{\gamma}_{1}=\bar{\gamma}_{2}$ and $\bar{\gamma}_{1}=2 \bar{\gamma}_{2}$, it reveals that the difference almost vanishes for $k=1$ while it is more obvious for $k=5$ and $k=14$.

The comparison of total outage capacity for BTB SCM and WTB SCM schemes is presented in Figure 10 for the 


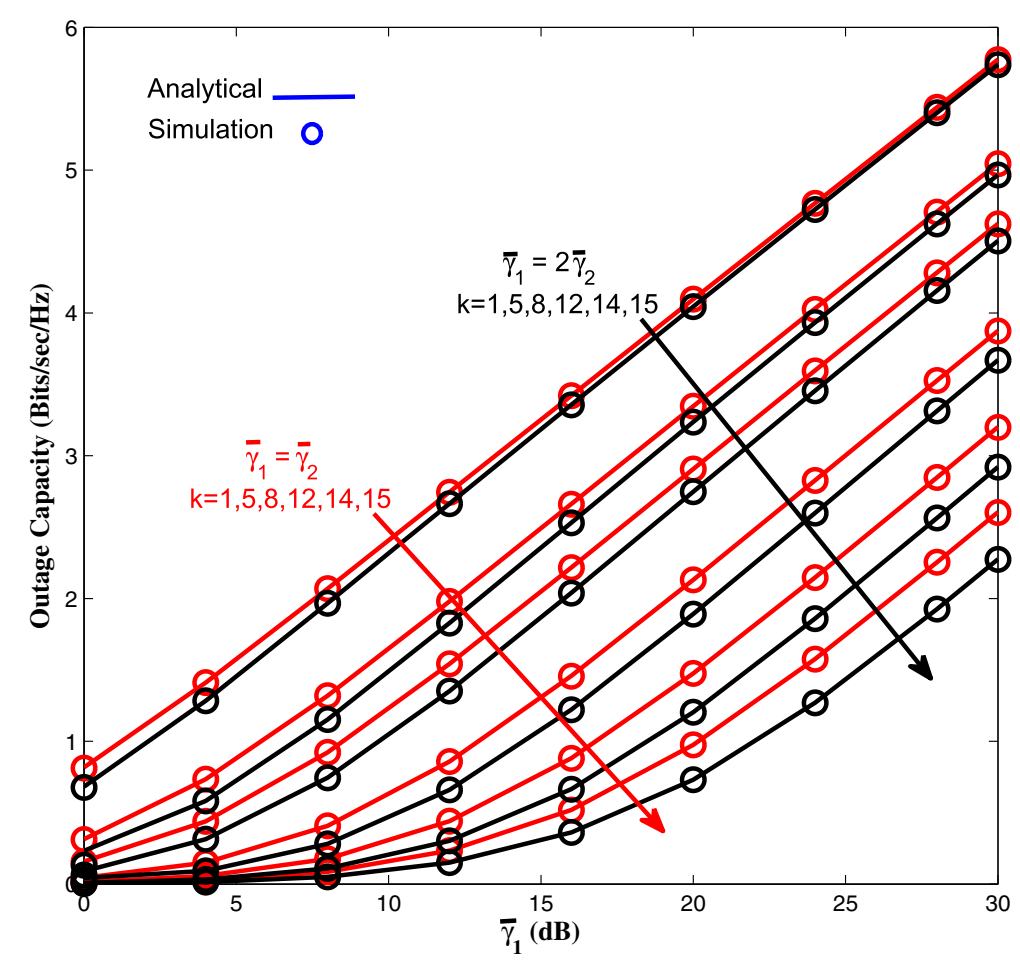

Figure 8 BTB SCM: $10 \%$ outage capacity comparison for $\bar{\gamma}_{1}=\bar{\gamma}_{2}$ and $\bar{\gamma}_{1}=2 \bar{\gamma}_{2}$.

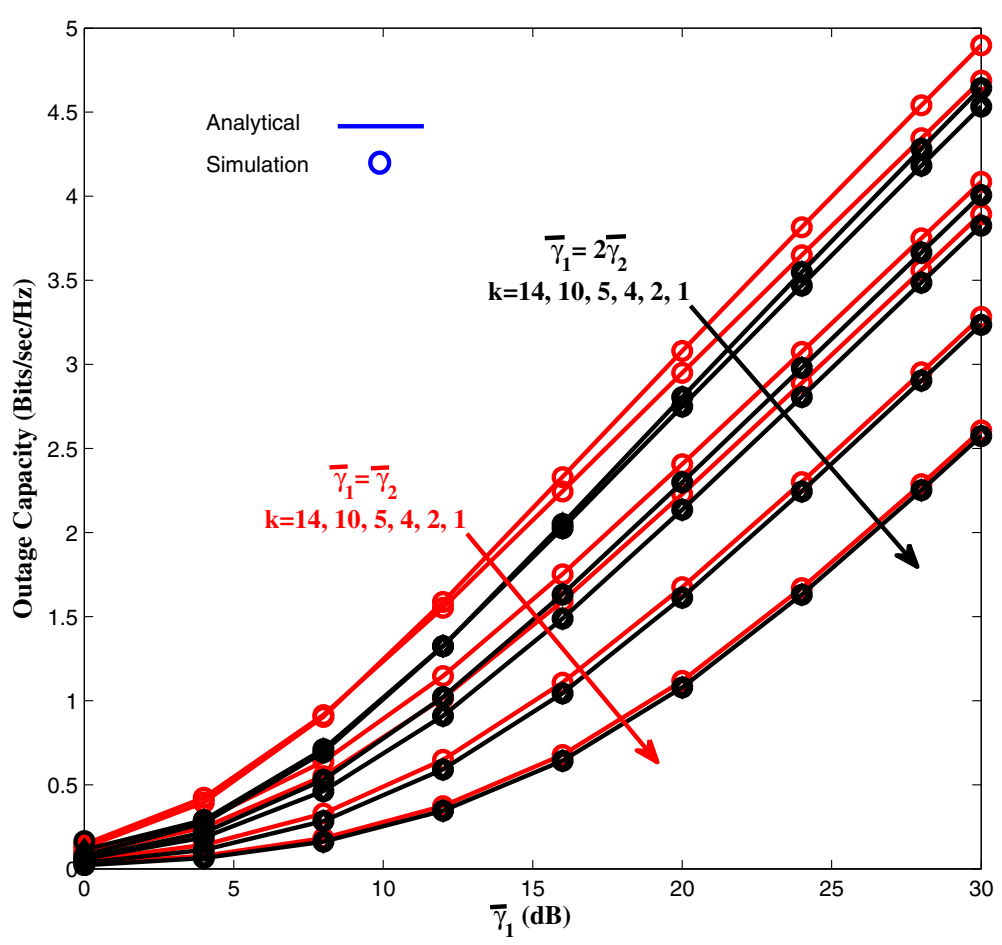

Figure 9 WTB SCM: $10 \%$ outage capacity comparison for $\bar{\gamma}_{1}=\bar{\gamma}_{2}$ and $\bar{\gamma}_{1}=2 \bar{\gamma}_{2}$. 


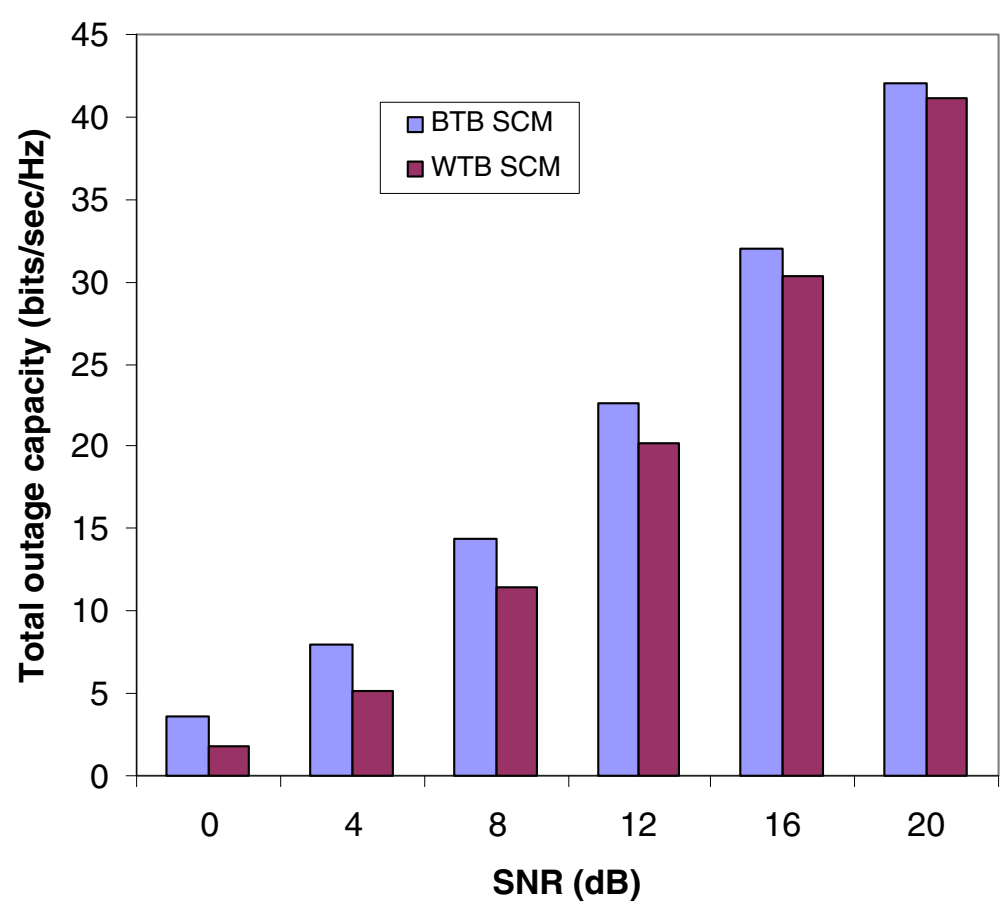

Figure 10 Comparison of BTB SCM and WTB SCM on the basis of outage capacity for $\bar{\gamma}_{1}=\bar{\gamma}_{2}$.

dual-hop OFDM relay system. It can be observed that BTB SCM outperforms WTB SCM on the basis of total outage capacity for the given range of SNR. It can be readily verified that at $\mathrm{SNR}=0,4,8 \mathrm{~dB}$, BTB SCM provide $206 \%$, $155 \%$, and $126 \%$ increase in outage capacity respectively as compared to WTB SCM. It shows that BTB SCM achieves significantly high outage capacity in lower and medium SNR regimes as compared to WTB SCM. For high SNR, i.e., $\mathrm{SNR}=16,20 \mathrm{~dB}$, the increase in outage capacity is $105 \%$ and $102 \%$, respectively. It is, therefore, concluded that BTB SCM outperforms WTB SCM on the basis of total outage capacity in all SNR regimes.

\section{Conclusions}

The outage performance of the dual-hop OFDM fixed gain AF relay system is analyzed in Rayleigh fading with BTB SCM and WTB SCM. The analysis is done on the basis of outage probability and outage capacity performances. Accurate close-form expressions for the outage probability and outage capacity are derived. The analysis shows that ordered SCM schemes results in categorization of subcarrier-pairs into best, moderate, and poor subsets of pairs. The BTB SCM scheme outperforms the WTB SCM scheme on the basis of outage probability for best and moderate sets of subcarrier pairs. The outage performance of poor set of subcarrier pairs is comparable in both schemes and show lower outage only in the high SNR regime. On the basis of outage capacity performance comparison, it is concluded that the BTB SCM scheme outperforms the WTB SCM scheme for all range of SNR. Specifically, in low and medium SNR regimes, a significant gain in outage capacity can be achieved. Furthermore, it is shown that the percentage increase in outage capacity due to BTB SCM decreases as the SNR increases.

\section{Appendix A}

Proof of outage probability expressions

The expression for $P_{\text {out }}^{k}$ in (5) can be rewritten as [29]

$$
P_{\text {out }}^{k}=\int_{0}^{\infty} \operatorname{Pr}\left(\frac{\gamma_{1}^{k} \gamma}{\gamma+\epsilon} \leq \gamma_{\text {th }}\right) f_{\gamma_{2}^{k}}(\gamma) d \gamma
$$

which can be expressed as the sum of two integrals as

$$
\begin{aligned}
P_{\text {out }}^{k}= & \int_{0}^{\gamma_{\text {th }}} \operatorname{Pr}\left(\gamma_{1}^{k}\left(\gamma-\gamma_{\text {th }}\right) \leq \epsilon \gamma_{\text {th }}\right) f_{\gamma_{2}^{k}}(\gamma) d \gamma \\
& +\int_{\gamma_{\text {th }}}^{\infty} \operatorname{Pr}\left(\gamma_{1}^{k}\left(\gamma-\gamma_{\text {th }}\right) \leq \epsilon \gamma_{\text {th }}\right) f_{\gamma_{2}^{k}}(\gamma) d \gamma
\end{aligned}
$$

It should be noted that the term $\operatorname{Pr}\left(\gamma_{1}^{k}\left(\gamma-\gamma_{\text {th }}\right) \leq \epsilon \gamma_{\text {th }}\right)$ in the first integral of (14) equals unity for $0 \leq \gamma \leq \gamma_{\text {th }}$, therefore (14) can be simplified as

$$
P_{\text {out }}^{k}=\int_{0}^{\gamma_{\text {th }}} f_{\gamma_{2}^{k}}(\gamma) d \gamma+\int_{\gamma_{\text {th }}}^{\infty} F_{\gamma_{1}^{k}}\left(\frac{\epsilon \gamma_{\text {th }}}{\gamma-\gamma_{\text {th }}}\right) f_{\gamma_{2}^{k}}(\gamma) d \gamma
$$


Using the identity $F_{X}^{\prime}(x)=1-F_{X}(x)$ where $F_{X}(x)=$ $\int_{-\infty}^{x} f_{X}(x) d x,(15)$ can be rewritten as

$$
P_{\text {out }}^{k}=1-\int_{\gamma_{\text {th }}}^{\infty} F_{\gamma_{1}^{k}}^{\prime}\left(\frac{\epsilon \gamma_{\text {th }}}{\gamma-\gamma_{\text {th }}}\right) f_{\gamma_{2}^{k}}(\gamma) d \gamma
$$

The change of variable $y=\gamma-\gamma_{\text {th }}$ allow us to rewrite (16) after some simple algebraic steps as

$$
\begin{aligned}
P_{\text {out }}^{k}= & 1-\int_{0}^{\infty} f_{\gamma_{2}^{k}}\left(y+\gamma_{\text {th }}\right) d y \\
& +\int_{0}^{\infty}\left(F_{\gamma_{1}^{k}}\left(\frac{\epsilon \gamma_{\text {th }}}{y}\right)\right) f_{\gamma_{2}}\left(y+\gamma_{\text {th }}\right) d y .
\end{aligned}
$$

where $F_{\gamma_{1}^{k}}(x) \in\left\{F_{\gamma_{1}^{k}}^{w}(x), F_{\gamma_{1}^{k}}^{s}(x)\right\}$. The symbols $F_{\gamma_{1}^{k}}^{w}(x)$ and $F_{\gamma_{1}^{k}}^{s}(x)$ denote the CDF of $k$ th weakest and $k$ th strongest subcarrier of hop-1, respectively. It remains to find the expressions for the PDF of $\gamma_{2}^{k}$ and the CDF of $\gamma_{1}^{k}$ to solve (17) for the close-form expression of $P_{\text {out }}^{k}$.

\section{WTB SCM}

For WTB SCM, (17) can be rewritten as

$$
\left(P_{\text {out }}^{k}\right)^{W T B}=1-I_{1}+I_{2}
$$

where $I_{1}=\int_{0}^{\infty} f_{\gamma_{2}^{k}}^{s}\left(y+\gamma_{\text {th }}\right) d y$ and $I_{2}=\int_{0}^{\infty}\left(F_{\gamma_{1}^{k}}^{w}\left(\frac{\epsilon \gamma_{\text {th }}}{y}\right)\right)$ $f_{\gamma_{2}}\left(y+\gamma_{\mathrm{th}}\right) d y$.

Using (3), the expression for $F_{\gamma_{1}^{k}}^{w}(\gamma)$ can be derived by solving a simple integral as

$$
F_{\gamma_{1}^{k}}^{w}(\gamma)=\sum_{i=0}^{k-1} \alpha_{i} \frac{1}{N-i}\left(1-e^{-\rho_{i} \gamma}\right) .
$$

Similarly, using (4), the PDF $f_{\gamma_{2}^{k}}^{s}\left(y+\gamma_{\text {th }}\right)$ can be expressed.

$$
f_{\gamma_{2}^{k}}^{s}\left(y+\gamma_{\mathrm{th}}\right)=\sum_{d=0}^{N-k} \alpha_{d} \lambda_{2} e^{-\rho_{d}\left(y+\gamma_{\mathrm{th}}\right)}
$$

Using (20), the integral $I_{1}$ can be simplified to

$$
I_{1}=\sum_{d=0}^{N-k} \alpha_{d} \frac{1}{N-d} e^{-\rho_{d} \gamma_{\mathrm{th}}}
$$

Using (19) and (20) allows us to write $I_{2}$ in the form of two integrals $I_{3}$ and $I_{4}$ as

$$
I_{2}=\sum_{i=0}^{k-1} \sum_{d=0}^{N-k} \alpha_{i} \alpha_{d} \lambda_{2} \frac{1}{N-i} e^{-\rho_{d} \gamma_{\text {th }}}\left[I_{3}-I_{4}\right]
$$

where $I_{3}=\int_{0}^{\infty} e^{-\rho_{d} y} d y$ and $I_{4}=\int_{0}^{\infty} e^{-\frac{\rho_{i} \epsilon \gamma_{\text {th }}}{y}-\rho_{d} y} d y$. The integral $I_{3}$ simplifies to

$$
I_{3}=\frac{1}{\rho_{d}}
$$

The integral $I_{4}$ can be solved using the identity ([30], eq. (3.471.9) as

$$
I_{4}=2 \sqrt{\frac{\rho_{i} \epsilon \gamma_{\mathrm{th}}}{\rho_{d}}} K_{1}\left(2 \sqrt{\rho_{i} \rho_{d} \epsilon \gamma_{\mathrm{th}}}\right)
$$

where $K_{1}(x)$ is the modified Bessel function of second kind. The integral $I_{2}$ can be expressed with the help of (23) and (24) as

$$
I_{2}=\sum_{i=0}^{k-1} \sum_{d=0}^{N-k} \alpha_{i} \alpha_{d} \frac{\lambda_{2}}{N-i} e^{-\rho_{d} \gamma_{\text {th }}}\left[\frac{1}{\rho_{d}}-2 \sqrt{\frac{\rho_{i} \epsilon \gamma_{\text {th }}}{\rho_{d}}} K_{1}\left(2 \sqrt{\rho_{i} \rho_{d} \epsilon \gamma_{\text {th }}}\right)\right]
$$

Substituting (21) and (25) into (18) allows us the write the close-form expression of outage probability for WTB SCM as (6).

\section{BTB SCM}

For BTB SCM, (17) can be rewritten as

$$
\left(P_{\text {out }}^{k}\right)^{\text {BTB }}=1-I_{1}+I_{5}
$$

where

$$
I_{5}=\int_{0}^{\infty}\left(F_{\gamma_{1}^{k}}^{s}\left(\frac{\epsilon \gamma_{\mathrm{th}}}{y}\right)\right) f_{\gamma_{2}}\left(y+\gamma_{\mathrm{th}}\right) d y .
$$

Using (4), the expression for the CDF of $k$ th strongest subcarrier of hop-1, i.e., $F_{\gamma_{1}^{k}}^{s}(\gamma)$ can be derived by solving a simple integral as

$$
F_{\gamma_{1}^{k}}^{s}(\gamma)=\sum_{i=0}^{N-k} \alpha_{i}^{\prime} \frac{1}{N-i}\left(1-e^{-\rho_{i} \gamma}\right)
$$

Using (20) and (27) allows us to write $I_{5}$ in the form of integrals $I_{3}$ and $I_{4}$ as

$$
I_{5}=\sum_{i=0}^{N-k} \sum_{d=0}^{N-k} \alpha_{i}^{\prime} \alpha_{d} \lambda_{2} \frac{1}{N-i} e^{-\rho_{d} \gamma_{\text {th }}}\left[I_{3}-I_{4}\right]
$$

The solutions for $I_{3}$ and $I_{4}$ from (23) and (24) respectively can be used to express $I_{5}$ as

$I_{5}=\sum_{i=0}^{N-k} \sum_{d=0}^{N-k} \alpha_{i}^{\prime} \alpha_{d} \frac{\lambda_{2}}{N-i} e^{-\rho_{d} \gamma_{\mathrm{th}}}\left[\frac{1}{\rho_{d}}-2 \sqrt{\frac{\rho_{i} \epsilon \gamma_{\mathrm{th}}}{\rho_{d}}} K_{1}\left(2 \sqrt{\rho_{i} \rho_{d} \epsilon \gamma_{\mathrm{th}}}\right)\right]$

Substituting (21) and (29) into (26) allows us to express the close-form expression of outage probability for BTB SCM as (7).

\section{Appendix B}

\section{Proof of outage capacity expression for WTB SCM}

To derive the close-form expression for outage capacity, the first and second moments of end-to-end SNR, i.e., $\mathbf{E}_{\gamma_{\text {end }}^{k}}^{\mathrm{WTB}}[\gamma]$ and $\mathbf{E}_{\gamma_{\text {end }}^{k}}^{\mathrm{WTB}}\left[\gamma^{2}\right]$ respectively are required for the 
WTB SCM scheme. The close-form expression of end-toend SNR for the dual-hop OFDM relay system with WTB SCM can be derived as $[9,13]$.

$$
f_{\gamma_{\text {end }}^{k}}^{\mathrm{WTB}}(\gamma)=\sum_{i=0}^{k-1} \sum_{d=0}^{N-k} \mathcal{B}_{i d} e^{-\rho_{i} \gamma}\left[\eta_{i d} \sqrt{\gamma} K_{1}(\xi(\gamma))+K_{0}(\xi(\gamma))\right]
$$

where $\mathcal{B}_{i d}=2 \alpha_{i} \alpha_{d} \lambda_{1} \lambda_{2} \epsilon, \quad \xi(\gamma)=2 \beta_{i d} \sqrt{\gamma}, \beta_{i d}=$ $\sqrt{\rho_{i} \rho_{d} \epsilon}$ and $\eta_{i d}=\sqrt{\frac{\rho_{i}}{\rho_{d} \epsilon}}$ and $K_{0}(x), K_{1}(x)$ are the modified Bessel functions of the second kind. The first moment $\mathbf{E}_{\gamma_{\text {end }}^{k}}^{\mathrm{WTB}}[\gamma]$ can be expressed as

$$
\begin{aligned}
\mathbf{E}_{\gamma_{\text {end }}^{K}}^{W T B}[\gamma]= & \int_{0}^{\infty} \gamma f_{\gamma_{\text {end }}^{k}}^{W T B}(\gamma) d \gamma=2 \mathcal{B}_{i d} \int_{0}^{\infty} \\
& \times\left[\eta_{i d} \gamma^{\frac{3}{2}} e^{-\rho_{i} \gamma} K_{1}(\xi(\gamma))+\gamma e^{-\rho_{i} \gamma} K_{0}(\xi(\gamma))\right] d \gamma
\end{aligned}
$$

The integrals in (31) can be solved with the help of ([30], eq. (6.643.3)) as

$$
\begin{aligned}
\mathbf{E}_{\gamma_{\text {end }}^{k}}^{\mathrm{WTB}}[\gamma]= & \sum_{i=0}^{k-1} \sum_{d=0}^{N-k} \mathcal{B}_{i d} e^{\rho_{d} \epsilon / 2} \frac{1}{\rho_{i}^{2} \rho_{d} \epsilon} \\
& \times\left[W_{-2, \frac{1}{2}}\left(\rho_{d} \epsilon\right)+\frac{1}{2} \sqrt{\rho_{d} \epsilon} W_{-\frac{3}{2}, 0}\left(\rho_{d} \epsilon\right)\right]
\end{aligned}
$$

where $W_{a, b}(x)$ denotes the confluent hyper-geometric function as defined in ([30], eq. (9.220.4)). The second moment $\mathbf{E}_{\gamma_{\text {end }}^{k}}^{\mathrm{WTB}}\left[\gamma^{2}\right]$ can be expressed as

$$
\begin{aligned}
\mathbf{E}_{\gamma_{\text {end }}^{k}}^{\text {WTB }}\left[\gamma^{2}\right]= & \int_{0}^{\infty} \gamma^{2} f_{\gamma_{\text {end }}^{k}}(\gamma) d \gamma=2 \mathcal{B}_{i d} \int_{0}^{\infty} \\
& \times\left[\eta_{i d} \gamma^{\frac{5}{2}} e^{-\rho_{i} \gamma} K_{1}(\xi(\gamma))+\gamma^{2} e^{-\rho_{i} \gamma} K_{0}(\xi(\gamma))\right] d \gamma
\end{aligned}
$$

which can be solved with the help of ([30], Equation (6.643.3)) as

$$
\begin{aligned}
\mathbf{E}_{\gamma_{\text {end }}^{k}}^{\mathrm{WTB}}\left[\gamma^{2}\right]= & \sum_{i=0}^{N-k} \sum_{d=0}^{N-k} 6 \mathcal{B}_{i d} e^{\rho_{d} \epsilon / 2} \frac{1}{\rho_{i}^{3} \rho_{d} \epsilon} \\
& \times\left[W_{-3, \frac{1}{2}}\left(\rho_{d} \epsilon\right)+\frac{1}{3} \sqrt{\rho_{d} \epsilon} W_{-\frac{5}{2}, 0}\left(\rho_{d} \epsilon\right)\right]
\end{aligned}
$$

Substituting (32) and (34) into (9) and (10) gives the first and second moments of ergodic capacity, i.e., $\mathbf{E}^{\mathrm{WTB}}\left[\mathrm{C}^{k}\right]$ and $\mathbf{E}^{\text {WTB }}\left[\left(C^{k}\right)^{2}\right]$ respectively for WTB SCM. Substituting these into (8) gives the expression for outage capacity which can be expressed in compact form as (11).

\section{Appendix C}

\section{Proof of outage capacity expression for BTB SCM:}

To derive the close-form expression for outage capacity the knowledge of first and second moments of end-to-end SNR, i.e, $\mathbf{E}_{\gamma_{\text {end }}^{k}}^{\mathrm{BTB}}[\gamma]$ and $\mathbf{E}_{\gamma_{\text {end }}^{k}}^{\mathrm{BBB}}\left[\gamma^{2}\right]$ respectively is required for the BTB SCM scheme. The PDF of $\gamma_{\text {end }}^{k}$ for the dualhop OFDM AF fixed gain relay system with BTB SCM can be derived as $[9,13]$

$f_{\gamma_{\text {end }}^{k}}(\gamma)=\sum_{i=0}^{N-k} \sum_{d=0}^{N-k} \tilde{\mathcal{B}}_{i d} e^{-\rho_{i} \gamma}\left[\eta_{i d} \sqrt{\gamma} K_{1}(\xi(\gamma))+K_{0}(\xi(\gamma))\right]$

where $\widetilde{\mathcal{B}}_{i d}=2 \alpha_{i}^{\prime} \alpha_{d} \lambda_{1} \lambda_{2} \epsilon$. The expressions for $\mathbf{E}_{\gamma_{\text {end }}^{k}}^{\mathrm{BTB}}[\gamma]$ and $\mathbf{E}_{\gamma_{\text {end }}^{\mathrm{K}}}^{\mathrm{BTB}}\left[\gamma^{2}\right]$ can be written as

$$
\begin{aligned}
\mathbf{E}_{\gamma_{\text {end }}^{k}}^{\mathrm{BTB}}[\gamma]= & \int_{0}^{\infty} \gamma f_{\gamma_{\text {end }}^{k}}(\gamma) d \gamma=2 \widetilde{\mathcal{B}}_{i d} \int_{0}^{\infty} \\
& \times\left[\eta_{i d} \gamma^{\frac{3}{2}} e^{-\rho_{i} \gamma} K_{1}(\xi(\gamma))+\gamma e^{-\rho_{i} \gamma} K_{0}(\xi(\gamma))\right] d \gamma
\end{aligned}
$$

and

$$
\begin{aligned}
\mathbf{E}_{\gamma_{\text {end }}^{k}}^{\mathrm{BTBB}}\left[\gamma^{2}\right]= & \int_{0}^{\infty} \gamma^{2} f_{\gamma_{\text {end }}^{k}}(\gamma) d \gamma=2 \widetilde{\mathcal{B}}_{i d} \int_{0}^{\infty} \\
& \times\left[\eta_{i d} \gamma^{\frac{5}{2}} e^{-\rho_{i} \gamma} K_{1}(\xi(\gamma))+\gamma^{2} e^{-\rho_{i} \gamma} K_{0}(\xi(\gamma))\right] d \gamma .
\end{aligned}
$$

respectively. The integrals in (36) and (37) can be solved with the help of identity ([30], eq. (6.643.3)), which can be expressed as

$$
\begin{aligned}
\mathbf{E}_{\gamma_{\text {end }}^{k}}^{B T B}[\gamma]= & \sum_{i=0}^{N-k} \sum_{d=0}^{N-k} \widetilde{\mathcal{B}}_{i d} e^{\rho_{d} \epsilon / 2} \frac{1}{\rho_{i}^{2} \rho_{d} \epsilon} \\
& \times\left[W_{-2, \frac{1}{2}}\left(\rho_{d} \epsilon\right)+\frac{1}{2} \sqrt{\rho_{d} \epsilon} W_{-\frac{3}{2}, 0}\left(\rho_{d} \epsilon\right)\right]
\end{aligned}
$$

and

$$
\begin{aligned}
\mathbf{E}_{\gamma_{\text {end }}^{k}}^{B T B}\left[\gamma^{2}\right]= & \sum_{i=0}^{N-k} \sum_{d=0}^{N-k} 6 \widetilde{\mathcal{B}}_{i d} e^{\rho_{d} \epsilon / 2} \frac{1}{\rho_{i}^{3} \rho_{d} \epsilon} \\
& \times\left[W_{-3, \frac{1}{2}}\left(\rho_{d} \epsilon\right)+\frac{1}{3} \sqrt{\rho_{d} \epsilon} W_{-\frac{5}{2}, 0}\left(\rho_{d} \epsilon\right)\right]
\end{aligned}
$$

respectively. Substituting (38) and (39) into (9) and (10) give the first and second moments of ergodic capacity, i.e., $\mathbf{E}^{\mathrm{BTB}}\left[C^{k}\right]$ and $\mathbf{E}^{\mathrm{BTB}}\left[\left(C^{k}\right)^{2}\right]$ respectively for BTB SCM. Substituting these into (8) gives the expression for outage capacity which can be expressed in compact form as (12). 


\section{Competing interests}

The authors declare that they have no competing interests.

\section{Acknowledgements}

This research is supported by Higher Education Commission (HEC) Pakistan, Asian Institute of Technology (AIT) Thailand, and National Institute of Informatics (NII), Japan.

\section{Author details}

${ }^{1}$ Department Telecommunications, AIT, Pathumthani, Thailand. ${ }^{2}$ Department of Communication Engineering, University of Oulu, Oulu, Finland. ${ }^{3}$ National Institute of Informatics (NII), Tokyo, Japan. ${ }^{4}$ Graduate University for Advanced Studies (SOKENDAI), Tokyo, Japan.

Received: 2 April 2014 Accepted: 20 November 2014 Published: 4 December 2014

\section{References}

1. C Cox, An Introduction to LTE: LTE, LTE-advanced, SAE and 4G Mobile Communications (John Wiley \& Sons, 2014)

2. X Tao, X Xu, Q Cui, An overview of cooperative communications. IEEE Commun. Mag. 50(6), 65-71 (2012)

3. S Abeta, in IEEE International Conference on Communication Systems (ICCS), Toward LTE commercial launch and future plan for LTE enhancements (LTE-advanced) (IEEE, 2010), pp. 146-150

4. G Kramer, M Gastpar, P Gupta, Cooperative strategies and capacity theorems for relay networks. IEEE Trans. Inf. Theory. 51(9), 3037-3063 (2005)

5. MO Hasna, M-S Alouini, in IEEE Transactions on Wireless Communications, A performance study of dual-hop transmissions with fixed gain relays, vol. 3, (2004), pp. 1963-1968

6. A Hottinen, T Heikkinen, in IEEE Conference on Inf. Sc. and Systems, Subchannel assignment in OFDM relay nodes, (2006), pp. 1314-1317

7. M Herdin, in IEEE Int. Conf. on Comm. A chunk based OFDM amplify-and-forward relaying scheme for $4 \mathrm{~g}$ mobile radio systems, vol. 10, (2006), pp. 4507-4512

8. T Riihonen, R Wichman, J Hamalainen, A Hottinen, in International ITG Workshop on Smart Antennas. WSA 2008. Analysis of subcarrier pairing in a cellular OFDMA relay link (IEEE, 2008), pp. 104-111

9. C Athaudage, M Saito, J Evans, in IEEE ICC, Performance analysis of dual-hop OFDM relay systems with subcarrier mapping, (2008), pp. 4419-4423

10. F Li, D Wang, in IEEE Wireless Communications and Networking Conference (WCNC). Performance analysis of cooperative OFDM network with ordered subcarrier pairing, (2011), pp. 1658-1663

11. T Riihonen, R Wichman, S Werner, Evaluation of OFDM (a) relaying protocols: capacity analysis in infrastructure framework. IEEE Trans. Vehicular Technol. 6(1), 360-374 (2012)

12. CK Ho, A Pandharipande, in IEEE VTC Spring, BER minimization in relay-assisted OFDM systems by subcarrier permutation, (2008), pp. 1489-1493

13. E Kocan, M Pejanovic-Djurisic, DS Michalopoulos, GK Karagiannidis, Performance evaluation of OFDM amplify-and-forward relay system with subcarrier permutation. IEICE Trans. Commun. 93(5), 1216-1223 (2010)

14. TWang, Y Fang, L Vandendorpe, Power minimization for OFDM transmission with subcarrier-pair based opportunistic DF relaying. IEEE Commun. Lett. 17(3), 471-474 (2013)

15. TWang, F Glineur, J Louveaux, L Vandendorpe, Weighted sum rate maximization for downlink OFDMA with subcarrier-pair based opportunistic DF relaying. IEEE Trans. Signal Process. 61(10), 2512-2524 (2013)

16. RA Shah, N Rajatheva, Y Ji, in IEEE International Conference on Communications (ICC), Analysis of BER and capacity for dual-hop OFDM relay system with subcarrier mapping in Nakagami-m fading (IEEE, 2014), pp. 5089-5094

17. JN Laneman, DN Tse, GW Wornell, Cooperative diversity in wireless networks: efficient protocols and outage behavior. IEEE Trans. Inf. Theory 50(12), 3062-3080 (2004)

18. MO Hasna, M-S Alouini, End-to-end performance of transmission systems with relays over Rayleigh-fading channels. IEEE Trans. Wireless Commun. 2(6), 1126-1131 (2003)
19. WP Siriwongpairat, AK Sadek, KR Liu, Cooperative communications protocol for multiuser OFDM networks. IEEE Trans. Wireless Commun. 7(7), 2430-2435 (2008)

20. M Kaneko, K Hayashi, P Popovski, K Ikeda, H Sakai, R Prasad, Amplify-and-forward cooperative diversity schemes for multi-carrier systems. IEEE Trans. Wireless Commun. 7(5), 1845-1850 (2008)

21. B Bai, W Chen, K Ben Letaief, Z Cao, in IEEE Global Telecommunications Conference (GLOBECOM). Outage exponent for OFDM channels, (2010), pp. 1-5

22. H Boostanimehr, VK Bhargava, Outage capacity analysis for OFDM decode-and-forward systems in frequency selective Rayleigh fading channels. IEEE Commun. Lett. 16(6), 937-940 (2012)

23. N Ksairi, in IEEE 8th International Conference on Wireless and Mobile Computing, Networking and Communications (WiMob), Outage performance analysis and optimization of OFDM-based relaying protocols, (2012), pp. 517-522

24. H Boostanimehr, V Bhargava, Outage probability analysis for multi-user single-relay OFDMA DF networks in frequency selective Rayleigh fading channels. IEEE Commun. Lett. 18(2), 245-948 (2014)

25. RA Shah, N Rajatheva, Y Ji, in IEEE International Conference on Communications Workshops (ICC), Outage probability and outage capacity analysis of cooperative OFDM system with subcarrier mapping (IEEE, 2014), pp. 314-319

26. J Pérez, J Ibánez, L Vielva, I Santamaria, Closed-form approximation for the outage capacity of orthogonal STBC. IEEE Commun. Lett. 9(11), 961-963 (2005)

27. S Jin, MR McKay, C Zhong, K-K Wong, Ergodic capacity analysis of amplify-and-forward mimo dual-hop systems. IEEE Trans. Inf. Theory. 56(5), 2204-2224 (2010)

28. SChen, W Wang, X Zhang, X Zhang, M Peng, $Y$ Li, in IEEE Wireless Communications and Networking Conference (WCNC), Ergodic and outage capacity analysis of amplify-and-forward mimo relay with OSTBCs, (2010), pp. 1-6

29. RH Louie, Y Li, B Vucetic, in IEEE International Conference on Communications (ICC), Performance analysis of beamforming in two hop amplify and forward relay networks, (2008), pp. 4311-4315

30. R Gradshteyn, Table of Integrals, Series, and Products (Academic Press, Orlando, Florida, 2007)

doi:10.1186/1687-1499-2014-209

Cite this article as: Shah et al:: Outage analysis of a dual-hop OFDM amplify-and-forward relay system with subcarrier mapping in Rayleigh fading. EURASIP Journal on Wireless Communications and Networking 2014 2014:209.

\section{Submit your manuscript to a SpringerOpen ${ }^{\circ}$ journal and benefit from:}

- Convenient online submission

- Rigorous peer review

- Immediate publication on acceptance

- Open access: articles freely available online

- High visibility within the field

- Retaining the copyright to your article

Submit your next manuscript at springeropen.com 